\title{
Radiological Aspect of Unusual Case of Rapidly Growing Giant Phyllodes Tumour of Breast
}

\section{ABSTRACT}

Phyllodes tumour or cystosarcoma phyllodes are a rare fibroepithelial tumour of breast, predominantly affecting the adult woman of age group 40-60 years. This is a case report of unusual, rapidly growing giant phyllodes in a 58-year-old women who came with history of enlarging lump in right breast without any significant pain or nipple discharge and was referred to the Radiology Department for mammographic and sonomammographic evaluation by the referring breast surgeon. This case is unique, since it describes how a rapidly growing breast lump is not always a sinister breast carcinoma but can be a benign, borderline or malignant phyllodes all of which can mimic a carcinoma in signs and symptoms, but hold a better prognosis. The final diagnosis of borderline phyllodes tumour was made on core biopsy followed by mastectomy of the involved breast. The main take away of this case report is how by having high level of suspicion, knowledge of specific imaging characteristics, correct biopsy technique combined with histopathology can help in reaching to a correct diagnosis leading to proper patient care and management.

Keywords: Fibroadenoma, Histopathology, Mammography, Sonomammography

\section{CASE REPORT}

A 58-year-old woman referred to Sonography Department with primary concern of rapidly growing painless mass in right breast with localised discomfort for six months. She had past history of previous lumpectomy in same breast without available reports or films for confirmation. No relevant previous medical or genetic history, no history of breast or ovarian carcinoma in mother or first degree maternal and paternal relatives. On physical examination, mass was hard on palpation with no associated skin thickening, nipple retraction, skin redness or inflammation, skin or nipple ulceration or oedema. In view of history of rapidly growing painless lump mammography, sonomammography and core biopsy was performed in the department followed by histopathology. No serum analysis was recommended, since imaging and histopathology provide the best possible diagnosis in such cases.

On digital mammography with breast tomosynthesis (HOLOGIC Selenia Dimension 3d), a non-specific large equal to high density lesion with lobulated, well circumscribed margin was identified occupying most of the right breast. A radiolucent halo was appreciated surrounding the mass. A small lucent centered calcification was noted in periphery of the mass lesion [Table/Fig-1,2].



[Table/Fig-1]: Craniocaudal view-Lobular marginated equal to high density lesion occupying most of right breast with associated skin thickening.

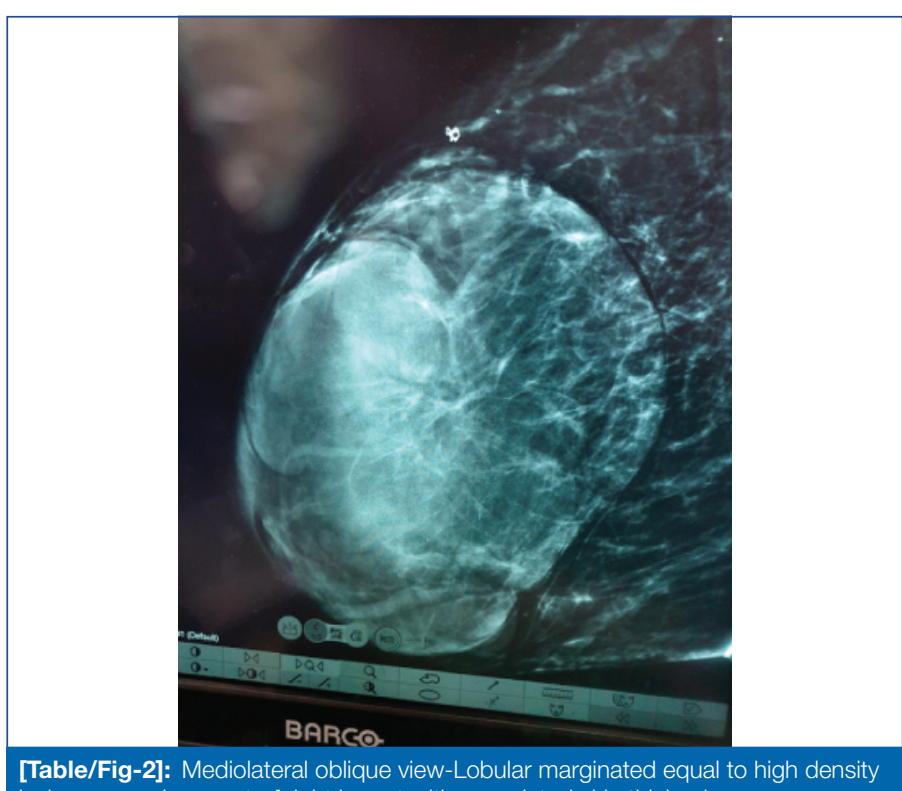

[Table/Fig-2]: Mediolateral oblique view-Lobular marginated equal to high density lesion occupying most of right breast with associated skin thickening.

On sonomammographic (performed on the same day as mammography) correlation using a linear probe (ML6-15-D, VOLUSON E10), a large iso to hypoechoic lobular marginated wider than taller solid mass lesion measuring approximately $11.1 \times 5.7 \times 9.0 \mathrm{~cm}$ was noted. Few cleft-like cystic spaces were seen within and in the periphery [Table/Fig-3-5]. On colour doppler interrogation, significant intralesional and peripheral vascularity was identified [Table/Fig-5]. No significant posterior acoustic characteristics were appreciated [Table/Fig-6]. In view of history of large, rapidly growing mass and significant vascularity on colour doppler, preliminary diagnosis of phyllodes tumour with Breast Imaging, Reporting and Data System (BI-RADS) category 4 was made. No suspicious lymph nodes were seen in bilateral axillae both on mammography and sonomammography.

In view of above imaging findings, a core biopsy was performed using 14 G BARD biopsy gun [Table/Fig-7] on the same day as mammography and samples were sent for histopathological analysis. After three days, the results revealed a concordant 
diagnosis of "Phyllodes Tumour-Borderline as per morphological features" with microscopic examination showing stromal proliferation with moderately cellular, intersecting fascicles of spindle cells with fairly bland nuclei are seen. Several elongated, mildly proliferative ductules lined by inner cuboidal and outer myoepithelial layer, periductal stromal condensation were seen with no heterogenous elements, pleomorphism or necrosis.

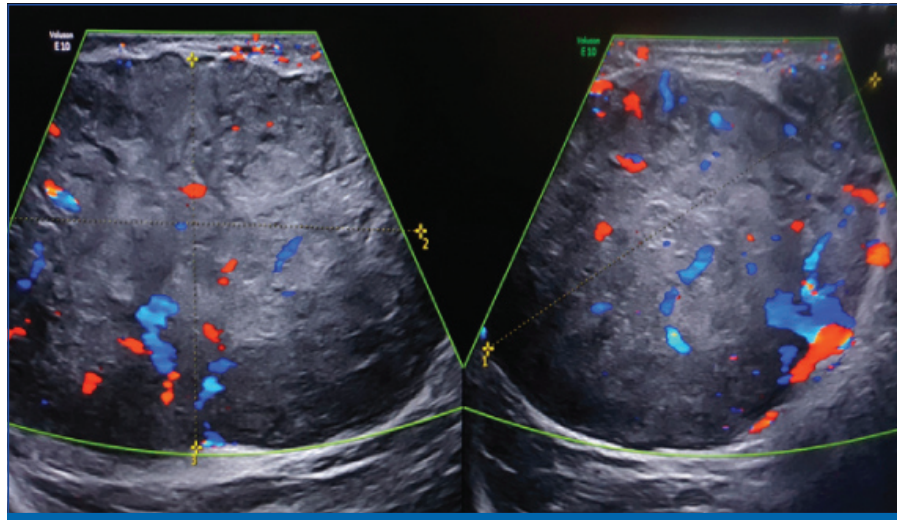

[Table/Fig-3]: Lobular margined heterogenous parallel lesion with cleft like cystic spaces and with significant intralesional and peripheral vascularity on Sonommaography.

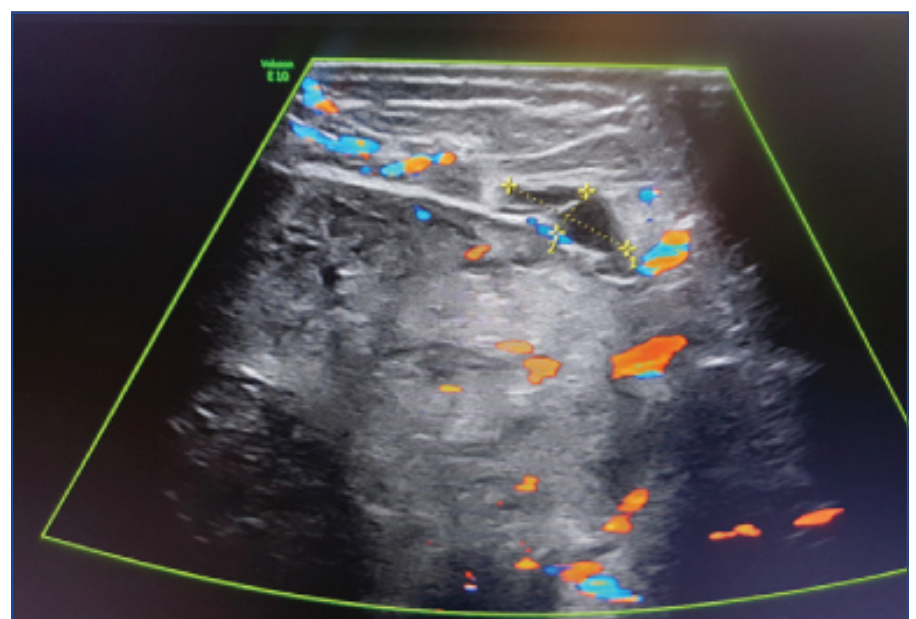

[Table/Fig-4]: Cystic spaces at the periphery of the mass lesion on

sonomammography.

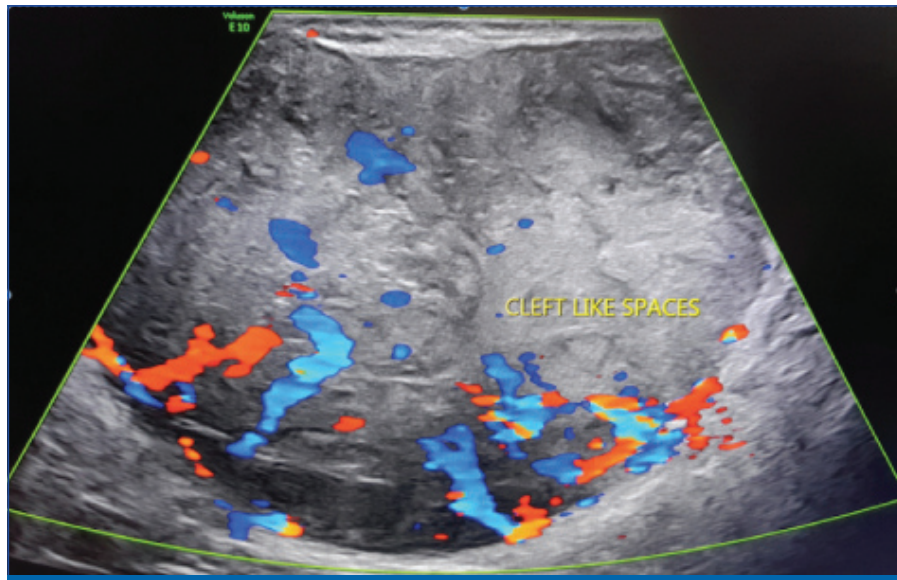

[Table/Fig-5]: Cleft like cystic spaces well seen with areas of increased vascularity on sonomammography.

In view of borderline phyllodes, after one week right mastectomy was performed in view of presurgical tests and imaging. Patient tolerated the procedure well with no adverse events and procedural and post procedural complications. Preventive care in terms of annual follow ups with mammography and sonomammography was advised. Self-breast examination as an early detector of future developing lump with no necessary pharmacological intervention was also recommended.
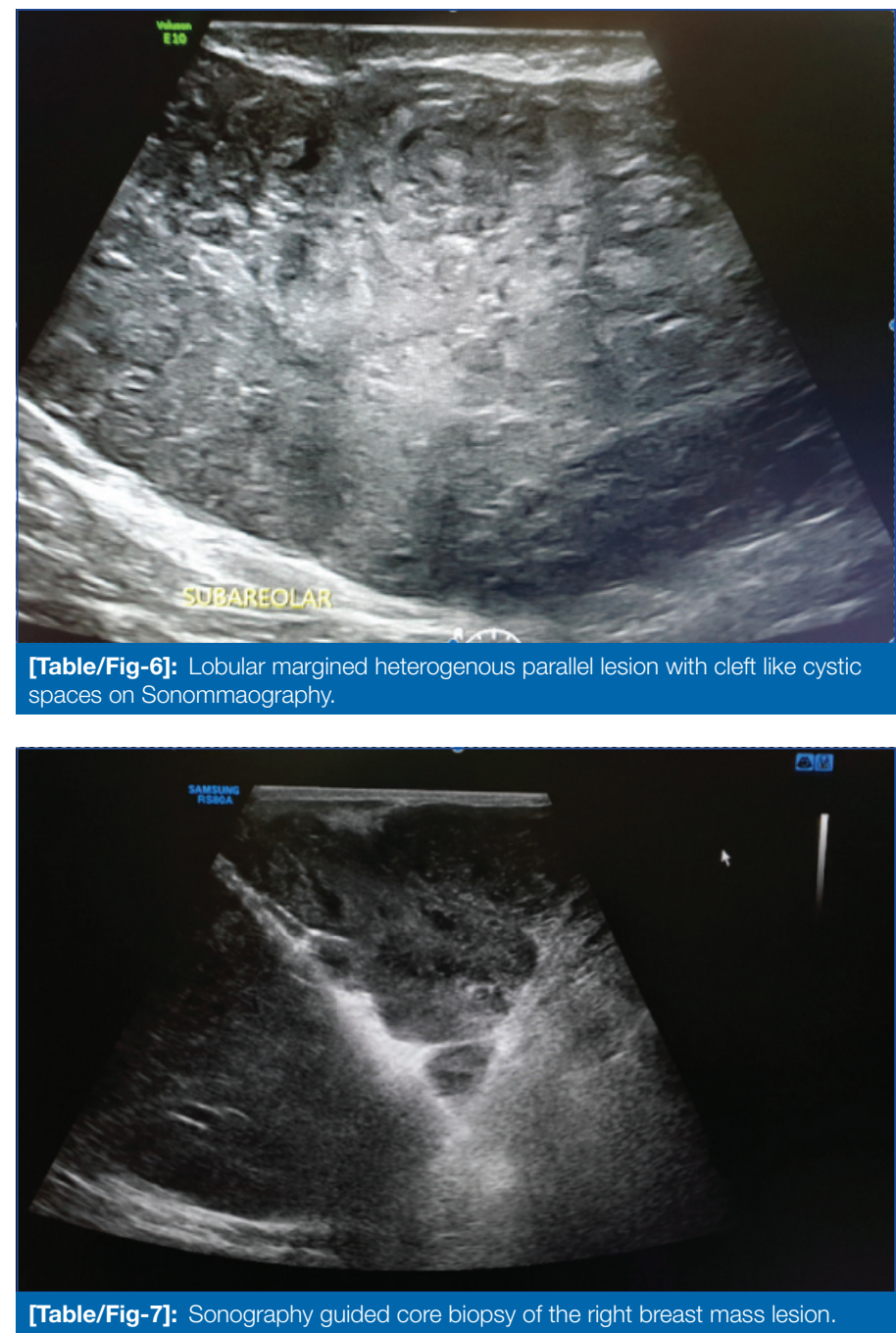

\section{DISCUSSION}

Phyllodes tumour or Cystosarcoma phyllodes are rare breast neoplasms which mostly appears during the $5^{\text {th }}$ or $6^{\text {th }}$ decade of life. It presents clinically as a large, benign-looking breast mass. However, it shows an unpredictable clinical behaviour [1]. Important factors which may be responsible for tumour growth are injury, breastfeeding, pregnancy and increased levels of oestrogen. The term phyllodes meaning "leaflike" refers to its histologic appearance in which a double layer epithelial component is surrounded by hypercellular stromal/mesenchymal component. In view of significant clinical and imaging similarities to more benign entity like fibroadenoma and at the same time overlapping with more sinister lesions namely primary sarcoma of breast and metaplastic carcinoma, correct diagnosis and treatment is of utmost importance.

On mammography, phyllodes tumour is typically seen as non-specific, large rounded oval or lobulated, generally well circumscribed lesions with smooth margins. A radiolucent halo may be present. Few may show coarse and plaque like calcification on mammogram [2]. On ultrasonography, these are benign-looking lesions with internal clefts, cystic spaces with moderate vascularity on colour doppler [3]. As described by Saraswat S and Kumar A in their study, they are rapidly growing tumours, with high recurrence rate and may even metastasize in rare cases [3]. They are seen as well defined, iso to hypoechoic lesions with well circumscribed margin, lobulated contour and posterior acoustic enhancement [4]. World Health Organization $(\mathrm{WHO})$ defines several histologic features that help differentiate phyllodes tumour from fibroadenomas classifying them into benign, borderline, or malignant depending on degree of atypia and overgrowth, stromal cellularity, mitotic count, tumour margin behaviour and the presence of malignant heterologous components [5]. Benign phyllodes tumours are generally categorised as BIRADS category 3 whereas aggressive phyllodes tumours are most 
categorised as category 4 [6]. As described by Yilmaz E et al., on histopathology they exhibit large slit-like spaces, cellular stroma which may be myxoid, with or without infiltrative border, mitosis, nuclear atypia and stromal overgrowth [7]. Differentiation between benign, borderline and malignant phyllodes is a histopathological diagnosis, though benign phyllodes tumours most commonly reveal heterogeneous echogenicity with multiple small anechoic clefts on sonography, whereas aggressive tumours were most commonly complex cystic and had solid echogenic appearance. The most common sonographic findings of benign phyllodes tumours is oval or round shaped lesion with circumscribed margin, heterogeneous echogenicity, absence of posterior acoustic features and increased vascularity. In aggressive phyllodes tumours, the most common sonographic findings are irregular shape, indistinct or microlobulated margin, complex cystic and solid appearance, posterior acoustic enhancement, and increased vascularity. The posterior acoustic features and location of vascularity does not differ significantly between benign and aggressive phyllodes tumours.

Fibroadenomas are usually seen in younger age group ranging from 21-40 whereas phyllodes tumour are usually more common in older age group, but significant overlap in imaging findings is not uncommon. Large size (4 $\mathrm{cm}$ or more), lobulated margin, high density and lucent halo sign on mammogram, lobular or irregular contour, intramural cleft like cystic areas, posterior acoustic enhancement are significantly more likely to be phyllodes tumours than fibroadenomas. Other differentials include breast sarcoma and metaplastic carcinoma. Breast sarcomas like angiosarcomas present with localised purple or blue discolouration of skin and can be multiple. In contrast, phyllodes tumour rarely presents with skin changes and are usually single. Fibrosarcomas are more commonly seen as spiculated breast mass in contrast to phyllodes which usually show circumscribed or macrolobulated margins. Metaplastic carcinomas are rare primary breast malignancy which closely resembles phyllodes on imaging. The final diagnosis can only be established by histopathology.

In present study, most of the findings are in concordance with other promised published literature. A case report published by Destounis SV et al., also shows a similar rapidly growing cystosarcoma phyllodes of breast which on mammography was seen as large well-circumscribed oval or lobulated mass and on sonography presented as solid well-defined masses with heterogeneous echoes similar to one seen in present study [8]. In an article published on phyllodes tumour by Parker SJ and Harries SA, local recurrence can usually be controlled by further wide excision and mastectomy is not invariably required. Mastectomy should, however, be considered for local recurrence after local surgery for borderline or malignant tumours, which was the main line of treatment in our patient, being a borderline histopathological subtype [9]. In a case report published by Paryani $\mathrm{J}$, et al., of a 50-year-old female with rapidly enlarging breast swelling since six months, who had a similar palpable breast lump as in present study patient, was managed with radical mastectomy with histopathology revealing encapsulated malignant phyllodes high grade tumour with mitotic rate of 32 per high-power field and clear margins. In present case study also, the management and histopathological findings were the same owing to the large size and borderline to malignant nature of the tumour [10].

The main strength of this case report is the correct patient management starting from clinical examination till mastectomy followed in a sequential manner in the institution, with coherence and good communication between radiological, histopathological and surgical teams which is of paramount importance for good patient care and management. The mammography and sonomammography was done by experienced radiologist with second and third review by other experienced radiologists in the department. All the clinical, diagnostic and surgical work up was done in the hospital premises which promises better patient's compliance and is convenient for the patient.

\section{CONCLUSION(S)}

Phyllodes tumour of breast is rare fibroepithelial tumours which show significant overlap with fibroadenomas, primary sarcomas and metaplastic breast carcinoma on imaging alone. The patient in this case study was satisfied with the diagnostic and surgical treatment received. The good prognosis of borderline phyllodes when caught in time is the advantage which adds on to the patient satisfaction and disease free period. Therefore proper history, imaging and timely histopathological evaluation play a relevant role in correct differentiation and diagnosis. However, high level of suspicion, knowledge of their imaging characteristics, correct biopsy technique combined with histopathology can help in reaching to a correct diagnosis leading to proper patient care and management.

\section{REFERENCES}

[1] Mazy S, Hustin J, Reepinghen PV. Phyllodes tumour of breast, case 923 Eurorad. 2001

[2] Muzo BD, Ceccilard F. Phyllodes tumour Radiopaedia 2008;1-3.

[3] Saraswat S, Kumar A. The study of different presentations of breast lumps in radiographic imaging. Acta Med Int 2014;1:45-51

[4] Bournias D, Chardali I, Iconomidis T, Prousalis A. Findings in mammography and sonomammography of Phyllodes tumour and fibroadenoma: Review article. Eurorad. 2006;1-9.

[5] Camara C, Gonzalez-Farre X, Vargas-Moniz J. Giant phyllodes tumour-Case report, Oncoplastic treatment and literature controversies. Revista de Seologia y Patologia Mamaria-Journal of Breast Science.2017;30(2):79-84.

[6] GW Shin, YM Park, JH Park, HJ Kim , HJ Choo, HJ Baek et al. Phyllodes Tumour of the Breast: Differentiation of Histological Grade by Ultrasonography. Hong Kong J Radiol. 2019;22:107-13

[7] Yilmaz E, Sal S, Lebe B. Differentiation of phyllodes tumours versus fibroadenomas. Acta Radiol. 2002;43(1):34-39.

[8] Destounis SV, Cortney Vogt AS, Arieno ALBS, Morgan RC. Difficult management of a rapidly growing Benign phyllodes tumour in a 49-year-old woman 2010;29(7):1125-31

[9] Parker SJ, Harries SA. Phyllodes tumours. Postgrad Med J. 2001;77(909):428-35.

[10] Paryani J, Gupta S, Chaturvedi A, Kumar V, Akhtar N, Aggarwal P et al. A giant malignant phyllodes tumour of the breast: A rare entity. Indian Journal of Gynecologic Oncology 2017;15(2):01-05.

PARTICULARS OF CONTRIBUTORS:

1. Consultant Radiologist, Department of Radiodiagnosis, Lilavati Hospital and Research Centre, Mumbai, Maharashtra, India.

2. Consultant Radiologist, Department of Radiodiagnosis, Lilavati Hospital and Research Centre, Mumbai, Maharashtra, India.

NAME, ADDRESS, E-MAIL ID OF THE CORRESPONDING AUTHOR:

Neha Subhash Tyagi,

801, Lalani Fairmont Building, $15^{\text {th }}$ Road, Khar West, Mumbai, Maharashtra, India. E-mail: nehatyagi17@gmail.com

\section{AUTHOR DECLARATION:}

- Financial or Other Competing Interests: None

- Was informed consent obtained from the subjects involved in the study? Yes

- For any images presented appropriate consent has been obtained from the subjects. Yes
PLAGIARISM CHECKING METHODS: [Jain Het al.]

- Plagiarism X-checker: Jul 26, 2020

- Manual Googling: Nov 28, 2020

- iThenticate Software: Jan 12, 2021 (13\%)
ETYMOLOGY: Author Origin

Date of Submission: Jul 22, 2020

Date of Peer Review: Sep 10, 2020

Date of Acceptance: Nov 28, 2020

Date of Publishing: Apr 01, 2021 